\title{
Traqueostomía quirúrgica abierta versus percutánea en pacientes con ventilación mecánica por COVID-19
}

\section{Tracheostomy surgical open versus percutaneous in patients on mechanical ventilation by COVID-19}

\author{
Ibzan J. Salvador-Ibarra ${ }^{1 *}$, Nancy V. Alva-Arroyo ${ }^{1}$, Alejandro Pizaña-Dávila ${ }^{1}$, José C. Gasca-Aldama², \\ Elizabeth L. Martínez-Hernández ${ }^{3}$ y Jorge E. Pérez-Figueroa ${ }^{3}$ \\ ${ }^{1}$ Unidad de Terapia Intensiva, Hospital Ángeles Mocel, Universidad Nacional Autónoma de México, Ciudad de México; ${ }^{2}$ Unidad de Terapia Intensiva, Hospital \\ Juárez de México, Universidad Nacional Autónoma de México, Ciudad de México; ${ }^{3}$ Unidad de Terapia Intensiva, Hospital Star Médica, Veracruz. México
}

\section{Introducción}

Desde que la Organización Mundial de la Salud declaró la enfermedad por coronavirus 2019 (COVID 19) una pandemia, la incidencia de síndrome de insuficiencia respiratoria aguda (SIRA) en pacientes hospitalizados con COVID-19 oscila entre el $17 \%$ y el $29 \%$, con una mortalidad del $3,4 \%^{1,2}$ y un promedio de 14.5 días desde el inicio de los síntomas hasta la ventilación mecánica ${ }^{3}$. En 1985, Ciaglia et al. describieron la técnica de la traqueostomía percutánea por dilatación (TPD) ${ }^{4}$. Actualmente, dos tercios de las traqueostomías se realizan en las unidades de cuidados intensivos ${ }^{5}$. Se ha demostrado que disminuye las complicaciones de la intubación prolongada, la resistencia y el espacio muerto en la vía aerea $^{6,7}$, la resistencia de la vía aérea y el espacio muerto. Los estudios muestran que la TPD es una alternativa a la traqueostomía quirúrgica abierta (TQA) $)^{8,9}$.

El objetivo del presente estudio fue describir las principales diferencias entre la TPD y la TQA en pacientes con COVID-19 en cuanto al tiempo para la realización del procedimiento y las complicaciones.

\section{Método}

Estudio prospectivo en dos centros hospitalarios de México, en pacientes hospitalizados en la unidad de cuidados intensivos con COVID-19, entre el 1 de abril de 2020 y el 31 de marzo de 2021. Las indicaciones para traqueostomía fueron ventilación mecánica prolongada definida como $>7$ días, índice de presión arterial de oxígeno/fracción inspirada de oxígeno ( $\mathrm{PaO} 2 / \mathrm{FiO} 2)$ que no respondió a posición prono, imposibilidad para mantener metas de protección pulmonar. Saturación por oximetría de pulso (SpO2), falla orgánica múltiple (FOM) y presión positiva al final de la espiración (PEEP) $\geq 10 \mathrm{cmH}_{2} \mathrm{O}$ o neumotórax. Se eliminaron los pacientes con coagulopatías y $\sin$ autorización.

\section{Análisis estadístico}

Los valores se expresaron como media y desviación estándar (DE) para las variables cuantitativas de distribución normal, y como mediana y rango intercuartilar para las variables cuantitativas de libre distribución; fueron sometidas a prueba de Shapiro-Wilk por la $\mathrm{N}=20$, y posteriormente se realizó un análisis bivariado, con significancia estadística establecida en $p<0.0050<5 \%$. Las variables categóricas se expresaron en número de observaciones y porcentajes. Se utilizó el programa SPSS versión 26.0.

\section{Correspondencia:}

*Ibzan J. Salvador-lbarra

E-mail: ibzjah@gmail.com

Disponible en internet: 07-03-2022

Fecha de recepción: 30-11-2021

Fecha de aceptación: 13-12-2021 DOI: 10.24875/REIE.21000223
Rev Educ Investig Emer. 2022;4(Supl 1):35-37 www.medicinadeemergencias.com bajo la licencia CC BY-NC-ND (http://creativecommons.org/licenses/by-nc-nd/4.0/). 
Tabla 1. Características demográficas de la población

\begin{tabular}{|c|c|c|c|}
\hline & Traqueostomía percutánea $(n=14)$ & Traqueostomía abierta $(n=6)$ & p \\
\hline \multirow[t]{2}{*}{ Género, n (\%) } & Femenino 2 (14) & Femenino 1 (23.8) & \multirow[t]{2}{*}{0.425} \\
\hline & Masculino 12 (85.7) & Masculino 5 (76.2) & \\
\hline Edad, años, mediana (RIC) & $55(46-62.2)$ & $57(41-63)$ & 0.312 \\
\hline IMC, $\mathrm{kg} / \mathrm{m}^{2}$, mediana (RIC) & $29(26-34.1)$ & $30.7(27-32.1)$ & 0.397 \\
\hline SOFA, mediana (RIC) & $8(7-9.7)$ & $7(6-9.6)$ & 1.09 \\
\hline APACHE II, media (RIC) & $19.5(14-25)$ & $18.6(15-24)$ & 0.841 \\
\hline $\begin{array}{l}\text { Comorbilidad, n (\%) } \\
\text { HAS } \\
\text { DM2 } \\
\text { Obesidad + ERC }\end{array}$ & $\begin{array}{c}4(26.7) \\
7(46.7) \\
3(20)\end{array}$ & $\begin{array}{l}3(42.9) \\
1(14.3) \\
2(28.6)\end{array}$ & $\begin{array}{c}0.062 \\
0.32 \\
1.04\end{array}$ \\
\hline
\end{tabular}

APACHE II: Acute Physiology And Chronic Health Evaluation II; DM2: diabetes mellitus tipo 2; ERC: enfermedad renal crónica; HAS: hipertensión arterial sistémica; IMC: índice de masa corporal; RIC: rango intercuartílico; SOFA: Sequential Organ Failure Assessment.

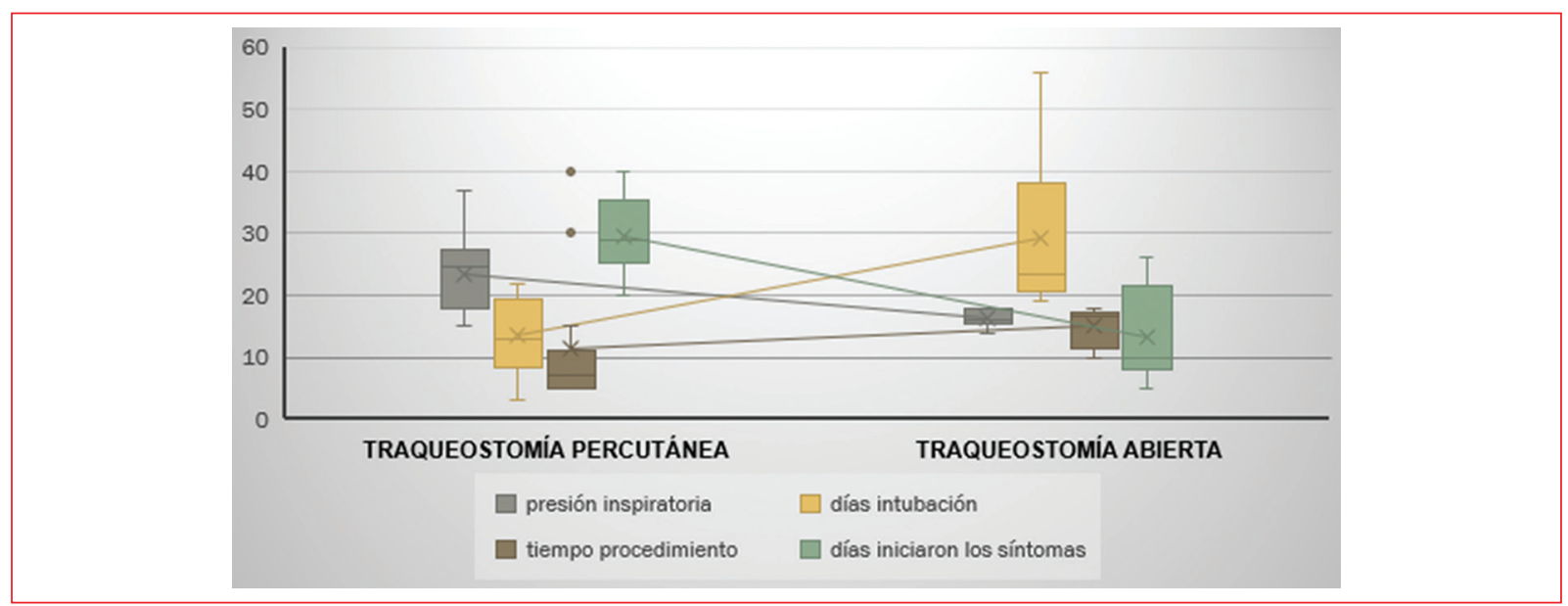

Figura 1. Comparación de los grupos de traqueostomía percutánea y traqueostomía abierta. Se encontraron diferencias con significancia estadística $(p<0.05)$ en cuatro variables: presión inspiratoria $(p=0.005)$, días de intubación $(p=0.002)$, tiempo de procedimiento $(p=0.026)$ y días en que se iniciaron los síntomas $(p=0.001)$.

\section{Resultados}

Se analizaron datos de 20 procedimientos: $14(70 \%)$ TPD y 6 (30\%) TQA. De sexo masculino fueron 12 (85.7\%) pacientes fueron del género masculino en el grupo de TPD y $5(76.2 \%)$ en el grupo de TQA. En el grupo de TPD predominó el sobrepeso y la obesidad en TQA, con una mediana de índice de masa corporal de 29 (rango: 26-34.1) y 30.7 (rango: 27-32.1) respectivamente (Tabla 1). Hubo más días de intubación en el grupo de TQA, 29 (DE: \pm 13.9 ), que en el grupo de TPD, 13.4 (DE: \pm 6.29$)$, con diferencia significativa $(p=0.002)$.
El tiempo de inicio de los síntomas hasta la realización de la traqueostomía fue de 29.5 días (rango: 25-35) para la TPD y de 13.3 días (rango: 8-21.5) para la TQA $(p=0.001)$. El tiempo para realizar el procedimiento fue menor para la TPD que para la TQA: 11.3 minutos (DE: \pm 7.0 ) vs. 15 minutos (DE: \pm 3.2 ) respectivamente, con significancia estadística $(p=0.026)$ (Fig. 1). No se observaron complicaciones intraoperatorias y solo tres pacientes presentaron sangrado posoperatorio leve, ocho pacientes sobrevivieron y seis fallecieron en el grupo de TPD; cuatro pacientes fallecieron y dos sobrevivieron en el grupo de TQA (Tabla 2). 
Tabla 2. índice de presión arterial de oxígeno/fracción inspirada de oxígeno (Pa02/FiO2)

\begin{tabular}{|c|c|c|c|c|c|}
\hline & \multicolumn{2}{|c|}{ Grupo traqueostomía percutánea } & \multicolumn{2}{|c|}{ Grupo traqueostomía abierta } & \multirow[t]{2}{*}{$\mathbf{p}$} \\
\hline & $n=14$ & IC95\% & $n=6$ & IC95\% & \\
\hline $\mathrm{SpO}_{2}$, mediana $(\mathrm{RIC})$ & $90.9(88-95)$ & $81-99$ & $93.6(92-96.5)$ & $91-99.3$ & 0.602 \\
\hline Vt, mediana (RIC), ml & $433(373-451)$ & $350.1-458$ & $446(410-470)$ & $390-485$ & 0.779 \\
\hline $\mathrm{FiO}_{2}$, media (DE) & $73.2( \pm 23.8)$ & $70-94.5$ & $57.5(48-68.7)$ & $42-71$ & 0.239 \\
\hline Presión inspiratoria, media (DE) & $23.4( \pm 6.02)$ & $21-29.6$ & $16.3(15.5-18)$ & $14.3-19.6$ & 0.005 \\
\hline $\mathrm{PaO}_{2} / \mathrm{FiO2}$ pretraqueostomía, mediana (RIC) & $113(95-132)$ & $93-133$ & $128.3(88-168)$ & $85-170$ & 0.239 \\
\hline 12 horas & $122(92.6-151)$ & $91.1-153$ & $105(83-132)$ & $84-130$ & 0.602 \\
\hline 24 horas & $118(91.3-145)$ & $91.6-148$ & $107(73-145)$ & $71-148$ & 0.779 \\
\hline 72 horas & $133(104-162)$ & $102-165$ & $103(49-156)$ & $43-160$ & 0.239 \\
\hline Días de estancia hospitalaria, mediana (RIC) & $20.3(14.2-26.5)$ & $13.1-27.2$ & $31(22.7-41)$ & $21-43$ & 0.076 \\
\hline Días de intubación, media (DE) & $13.4( \pm 6.29)$ & $8.25-19.5$ & $29( \pm 13.9)$ & $20-38$ & 0.002 \\
\hline Tiempo de procedimiento, minutos & $11.3( \pm 7.0)$ & $5-11.25$ & $15( \pm 3.2)$ & $11.5-17.2$ & 0.026 \\
\hline Días de inicio de los síntomas, mediana (RIC) & $29.5(25-35)$ & $19-36$ & $13.3(8-21.5)$ & $6.7-22.5$ & 0.001 \\
\hline Complicaciones intraoperatorias y posoperatorias & 2 & & 1 & & \\
\hline
\end{tabular}

DE: desviación estándar; $\mathrm{FiO}_{2}$ : fracción inspirada de oxígeno; IC95\%: intervalo de confianza del 95\%; PaO 2: presión arterial de oxígeno; RIC: rango intercuartílico; $\mathrm{SpO}_{2}$ : saturación por oximetría de pulso; Vt: volumen tidal.

\section{Conclusiones}

La TPD a la cabecera del paciente dentro de los primeros 14 días de intubación es un procedimiento seguro, rápido y con bajo riesgo de complicaciones para el paciente.

\section{Financiamiento}

Los autores no recibieron financiamiento para llevar a cabo este artículo.

\section{Conflicto de intereses}

Los autores declaran no tener conflictos de intereses.

\section{Responsabilidades éticas}

Protección de personas y animales. Los autores declaran que para esta investigación no se han realizado experimentos en seres humanos ni en animales.

Confidencialidad de los datos. Los autores declaran que han seguido los protocolos de su centro de trabajo sobre la publicación de datos de pacientes.
Derecho a la privacidad y consentimiento informado. Los autores han obtenido el consentimiento informado de los pacientes y/o sujetos referidos en el artículo. Este documento obra en poder del autor de correspondencia.

\section{Bibliografía}

1. World Health Organization. WHO-AUDIO Emergencies Coronavirus Press Conference 03 March 2020. (Consultado el 29-03-2020.) Disponible en: https://www.who.int/docs/default-source/coronaviruse/transcripts/who-audio-emergencies-coronavirus-press-conference-full-03mar2020-final.pdf

2. Chen N, Zhou M, Dong X, Qu J, Gong F, Han Y, et al. Epidemiological and clinical characteristics of 99 cases of 2019 novel coronavirus pneumonia in Wuhan, China: a descriptive study. Lancet. 2020;395:507-13.

3. Zhou F, Yu T, Du R, Liu Y, Liu Z, Xiang J, et al. Clinical course and risk factors for mortality of adult inpatients with COVID-19 in Wuhan, China: a retrospective cohort study. Lancet. 2020;395:1054-62.

4. Ciaglia P, Firsching R, Syniec C. Elective percutaneous dilatational tracheostomy. A new simple bedside procedure; preliminary report. Chest. 1985;87:715-9.

5. Zeitouni AG, Kost KM. Tracheostomy: a retrospective review of 281 cases. J Otolaryngol. 1994;23:61-6.

6. Barry BN, Bodenham AR. The role of tracheostomy in ICU. Anaesth Intensive Care Med. 2004;5:375-8.

7. Gosling AF, Bose S, Gomez E, Parikh M, Cook C, Sarge T, et al. Perioperative considerations for tracheostomies in the era of COVID-19. Anesth Analg. 2020;131:378-86.

8. Indeck M, Peterson S, Smith J, Brotman S. Risk, cost, and benefit of transporting ICU patients for special studies. J Trauma. 1988:28:1020-5.

9. Heikkinen M, Aarnio P, Hannukainen J. Percutaneous dilational tracheostomy or conventional surgical tracheostomy? Crit Care Med. 2000;28:1399-402. 